Revista Educación 25(2):49-58 2001

\title{
PROFESIONALES DE ORIENTACIÓN Y LA PERSPECTIVA DE GÉNERO EN COSTA RICA
}

Cecilia Villarreal Montoya

Resumen: Este artículo presenta los resultados de una investigación cualitativa, realizada con un grupo de profesionales de Orientación en servicio, sobre los alcances de la incorporación de la perspectiva de género en el quehacer orientador.

La condición de género hace referencia y cuestiona la determinación política, económica y sociocultural para el ser mujer-femenina y hombre-masculino, y además replantea las relaciones e interpretaciones inter e intragenéricas en la convivencia humana. Estos aspectos no pueden dejarse por fuera en la intervención orientadora que busca la clarificación y el replanteamiento del sentido de vida como una tarea del desarrollo bumano.

El grupo de profesionales, que participa con sus reflexiones y experiencias laborales, concuerda en la necesidad de tomar en cuenta la realidad genérica de las poblaciones con que trabajan. En su mayoria, muestran claridad en cuanto a los conceptos género, inequidad genérica, igualdad de oportunidades, y además desean conocer más sobre la implementación de la perspectiva de género en la acción profesional.

Los resultados de esta investigación, sin duda alguna, son un insumo importante para realimentar la formación y la actualización de profesionales en este campo.

\section{Introducción}

La perspectiva de género ha ido permeando la producción de conocimiento, el quehacer profesional de muchas disciplinas y el funcionamiento de diversas instituciones sociales. La educación y el sistema educativo no son la excepción. Una muestra de ello, en Costa Rica, es el esfuerzo de la coordinación que ha realizado el Ministerio de Educación Pública (MEP) con otras instancias para poner en práctica proyectos específicos que propician la democracia genérica. Entre ellos se encuentran los siguientes: 1. Implementación de políticas de género en Colegios Técnicos Profesionales y Académicos con valor agregado (1996). 2. Construcción de una cultura equitativa de género en la Escuela Líder (1988). 3. Plan de Acción Estratégico del Sistema Educativo Nacional: Hacia una Cultura de Equidad de Género (1999).

La puesta en marcha de estos proyectos tiene, inevitablemente, implicaciones disciplinarias y profesionales de las áreas del conocimiento que forman parte de la propuesta curricular del MEP. Además, el éxito que se obtenga depende, en gran medida, de la formación y de la actitud del personal docente y administrativo responsable del desarrollo de estos proyectos.

No se puede obviar que al asumir responsabilidades laborales, cada profesional adopta una posición ideológica, filosófica y 
teórico-práctica, y desde allí brinda sus aportes en cuanto al tipo de sociedad a la que aspira. La acción laboral refleja tanto los conocimientos como las creencias y las actitudes que hombres y mujeres quieren reproducir; "en definitiva, lo que hacemos tiene mucho que ver con lo que somos" (Rivas, 1995, p. 26). Y lo que somos refleja las experiencias de vida y la formación recibida para la producción de conocimiento y el desempeño de funciones específicas en un campo de estudio en particular. Por ello, los procesos de actualización de las disciplinas y de las profesionales y los profesionales que las ponen en práctica, deben ser una tarea permanente.

Dentro de este espíritu, este artículo analiza la posición de un grupo de profesionales en Orientación de la Región Educativa de San Carlos sobre la incorporación de la perspectiva de género en su desempeño laboral. Para lo cual fue necesario profundizar en los siguientes aspectos:

1. ¿Cómo concibe este grupo de profesionales la Orientación?

2. ¿Con qué nivel de formación e información sobre género cuenta este grupo de profesionales, y cómo ha sido adquirida?

3. ¿Qué comprensión tiene este grupo sobre la perspectiva de género y cómo la utilizan en la acción profesional?

4. ¿Qué condiciones consideran necesarias para incorporar la perspectiva de género en la labor profesional?

Para dar respuesta a estas interrogantes se realizó un acercamiento cualitativo de investigación el cual permitió recoger las ideas, creencias, percepciones y experiencias del grupo de profesionales participantes con respecto a la temática de interés.

\section{Acercamiento teórico}

La Orientación contribuye a que las personas tengan una amplia comprensión de sí mismas, de la realidad contextual y natural donde viven, y del sentido que les permite cumplir con su misión vital.

En otras palabras, que hombres y mujeres tengan claridad y sentido de responsabilidad sobre: ¿Cómo experimentan y asumen la vida? ¿Con qué fines desean lograr el mejor desarrollo de las propias potencialidades? ¿Cuál es la misión que cada quien puede cumplir en la vida?

El sentido de vida recoge el significado concreto que para cada persona tiene su existencia, así como los principios en los cuales se apoya y le permiten enfrentar los diferentes momentos y situaciones (Frankl, 1984). Para Corey (1990), el ser humano se distingue fundamentalmente por la sensación de significado y el tener propósitos de vida ya que el tener una misión personal es esencial. Por ello, para este mismo autor, cualquier persona sin un objetivo de vida, su vida estará vacía, se sentirá insatisfecha y si esos sentimientos se agudizan se sentirá decepcionada, angustiada, deprimida y tendrá síntomas que no son, sino del hecho de vivir una vida externamente dirigida. No es casual; entonces, que la mayor fuente de estrés y ansiedad humana, tiene como origen la confusión o la poca claridad sobre qué se quiere y sobre todo qué se persigue en los diferentes momentos de la vida (Frankl, 1984).

La naturaleza humana conlleva la búsqueda de ubicación consciente en el tiempo y en el espacio para la consecusión de la plenitud personal en interrelación productiva con la naturaleza y la colectividad. Sin embargo, el cómo cada persona se conciba a sí misma y a su propia vida definirá en gran medida sus metas y tareas vitales, así como sus responsabilidades y expectativas de vida, las cuales serán reflejadas en la actuación cotidiana.

Los procesos educativos en general transmiten y entrenan a las mujeres y a los hombres en cuanto a las expectativas que la sociedad tiene para ambos. Y de esta forma es que el sentido de vida colectivo-genérico determina los significados y las metas de vida individuales según el género. Este se refiere al cómo la persona se conciba a sí misma en 
términos femeninos y masculinos (Wood, 1999). Una vez que se adquiere la identidad genérica (que se da antes de adquirir la identidad sexual), cuando el niño se asume como masculino y la niña como femenina, ésta se convierte en un tamiz por el que pasan todas las experiencias de vida (Lamas, 1996).

Y es que la femineidad y la masculinidad contienen las creencias que definen funciones y roles sociales diferenciados para hombres y para mujeres, sin que éstos tengan una relación directa con las características sexuales y anatómicas (Fernández, 1998). En este sentido es que, tanto la masculinidad como la femineidad, son construcciones sociales y por lo tanto son suceptibles al cambio y pueden variar en diferentes culturas y épocas históricas.

En síntesis, el sentido de vida se va construyendo y replanteando en la interacción dialéctica de los hombres y las mujeres consigo mismas, de la relación entre ambos, de ellos y ellas con la sociedad y con la naturaleza. Este no es un proceso fácil ni lineal; por el contrario, está lleno de contradicciones y clama por la toma constante de decisiones simples y complejas.

La sociedad se apoya en sus propias instituciones (familia, sistema educativo, jurídico, ideológico; los medios de comunicación, etc.) para reproducir y mantener este sistema de inequidad, de desigualdad y de opresión y violencia de quienes están en mejores condiciones o gozan de mayores cuotas de poder, por sobre quienes no las tienen.

Las consecuencias de esta situación se pueden observar en los índices de pobreza, el analfabetismo, la deserción escolar, el desempleo, la inseguridad social, la problemática familiar y social, la desigualdad del desarrollo de grupos humanos (mujeres, grupos raciales etc.) y de zonas geográficas. Si se quiere concretar en números esta realidad para Costa Rica, se puede revisar el Informe del Estado de la Nación de los últimos años.

Esta situación sobrepasa las fronteras de los países, pudiéndose ver relaciones parecidas de dominación cultural de unas naciones por otras, y competencia económica entre ellas, lo que da como resultado bloques enriquecidos y con dominio por sobre los empobrecidos.

La relación individuo-sociedad es dual, pues la persona influye en su medio ambiente al mismo tiempo que éste influye en la persona (Hewitt, 1988). Esta relación se se presenta haciendo uso de la simbología social y de los significados aprendidos en la interacción social.

Esta no es una relación pasiva ya que, por un lado, las miembras y los miembros de la sociedad, son también seres libres, pensantes y actuantes y, por lo tanto transformadores. Se caracterizan por la capacidad de desarrollar la autoconciencia y por la utilización de símbolos (Hewitt, 1988).

Las personas son capaces de interpretar indicaciones de otras personas, al mismo tiempo que pueden establecer comunicación consigo mismas como con los demás. Al pensar, sentir, experimentar emociones, definir objetivos y propósitos, se está en condiciones de tomar decisiones, hacer planes, y realizar cambios significativos durante el proceso de vida.

En este sentido es que Rogers (en Campos, 1982), plantea que los seres humanos cuentan con las condiciones propias para crecer y desarrollarse como personas. Esto implica el desarrollo máximo de las potencialidades físicas, intelectuales, emocionales, espirituales y de interacción.

Desde la perspectiva del interaccionismo simbólico, las personas están construidas por un "sí mismas", que se entiende como la capacidad para pensar, imaginar, planear, reflexionar, decidir y juzgar, y por "una mente", la capacidad para interpretar significados, el proceso por medio del cual cada quien conversa consigo mismo sobre el significado de la situación. Los procesos de socialización inducen a hombres y mujeres a realizar estas actividades de forma diferenciada y en condiciones desiguales.

En este sentido, la conducta de las personas es determinada tanto por la autoafirmación como por la definición de roles designados 
socialmente (Franklin,1883). La cultura patriarcal define los roles, las funciones y el comportamiento de hombres y mujeres según los estereotipos construidos alrededor de la designación genérica y según las etapas del desarrollo humano existe un "deber ser" genérico.

$\mathrm{Al}$ actuar hay una conducta encubierta y una conducta manifiesta (Ritzer,1983). La conducta encubierta tiene que ver con el proceso mental que involucra la simbología y el significado respecto a la situación, la capacidad reflexiva. Para Mead (en Ritzer, 1983), los procesos internos deben entenderse como una fase de los procesos sociales, lo cual tiene que ver con el sí misma y la mente de las personas en las situaciones específicas. La conducta manifiesta es precisamente la actuación que se puede observar. La actuación de la persona que muestra la resolución que logró hacer en el acto reflexivo sobre lo percibido y lo definido en la situación específica y en las condiciones específicas.

Desde esta postura se puede concluir, entonces, que la conjugación de la individualidad con la colectividad, para las mujeres y los hombres, es un acto constante, profundo y que necesariamente se debe vivir con responsabilidad personal. Esto implica la comprensión individual de los significados sociales como un paso necesario para el planteamiento y replanteamiento de los propósitos de vida.

\section{La perspectiva de género en la acción profesional}

Como ya se planteó en el apartado anterior, el contexto sociocultural determina la "normativa" sobre el comportamiento aceptado para hombres y mujeres. La comprensión de esa situación permite a las personas tomar posición con respecto a esa realidad. O lo que es lo mismo, poder vivir conscientemente tal y como lo define Branden (1991), para quien esta condición se logra al asumirse la responsabilidad del conocimiento adecuado a la acción que se realiza. Entonces, el vivir conscientemente implica estimular la autocriticidad y la reflexión de la actuación humana, además de que se plantea la posibilidad de cambio.
El hecho de nacer hombre o mujer marca necesariamente la experiencia de vida de cada quien. Esta situación se da porque cada sociedad define las categorías genéricas cognitivas, ellas son las calificaciones, clasificaciones, y la jerarquización de lo masculino y lo femenino (Hériter,1996). Y la adquisición individual de esta categorización se logra por medio de procesos de aprendizaje en la familia, el sistema de educación formal e informal, el sistema jurídico e ideológico, los medios de comunicación, entre otros. En el aprendizaje social que ofrecen estas instituciones se conjugan procesos sicológicos, cognitivos, emocionales y motivacionales que aseguran la reproducción de la cultura patriarcal, porque tanto las mujeres como los hombres asimilan los mismos aprendizajes.

Para Castells (1999), el patriarcado actualmente es la estructura básica de todas las sociedades. "Este se caracteriza por la autoridad, impuesta desde las instituciones, de los hombres sobre las mujeres y sus hijos en la unidad familiar. Para que se ejerza esta autoridad, el patriarcado debe dominar toda la organización de la sociedad, de la producción y el consumo a la política, el derecho y la cultura. Las relaciones interpersonales $y$, por tanto, la personalidad, están bien marcadas por la dominación y la violencia que se originan en la cultura y las instituciones del patriarcado"(Castells, 1999, p. 159).

La "condición genérica" hace referencia y cuestiona la determinación política, económica y sociocultural para el ser mujer-femenina y hombre-masculino, y además replantea las relaciones intra e interpersonales e inter e intragenérica en la convivencia humana.

La "perspectiva de género" es un aporte científico del feminismo que sintetiza la teoría de género y una filosofía liberadora que busca dilucidar "¿Por qué la diferencia sexual implica desigualdad social?" (Lamas,1996, p.13). Esta perspectiva, según Lagarde (1997), analiza las posibilidades vitales de las mujeres y de los hombres como: el sentido de sus vidas, sus expectativas y oportunidades, las complejas y diversas relaciones 
sociales que se dan entre ambos géneros, así como los conflictos institucionales y cotidianos que deben enfrentar y las maneras en que lo hacen. También permite contabilizar los recursos y las capacidades de acción con que cuentan mujeres y hombres para enfrentar las dificultades de la vida y la realización de sus propósitos. Según esta misma autora, a un nivel macrosocial esta perspectiva permite correlacionar las posibilidades de vida de ambos géneros con el tipo de sociedad, el desarrollo histórico, la diversidad cultural y los modelos de desarrollo de la humanidad. En una forma muy abreviada, la perspectiva de género "tiene como uno de sus fines contribuir a la construcción subjetiva y social de una nueva configuración a partir de la resignificación de la historia, la sociedad, la cultura y la política desde las mujeres y con las mujeres" (Lagarde, 1997, p. 13).

Para tener una comprensión global de la historia es necesario rescatar el protagonismo de las mujeres, que se ha tendido a invisibilizar por la historiografía tradicional. Y además, se debería replantear y redimensionar la masculinidad y la hegemonía masculina.

La perspectiva de género estimula inevitablemente la toma de conciencia sobre la vivencia subjetiva y objetiva de mujeres y hombres en la sociedad patriarcal. Este no es un proceso fácil ni necesariamente placentero, por el contrario, es un cambio profundo que si bien es cierto es doloroso, también es liberador en cuanto permite la comprensión de la historia de la humanidad y de las mujeres y los hombres en particular. Esta toma de conciencia, es un descubrimiento esperanzador ya que al no parecer natural ni divina la discriminación y las relaciones asimétricas y de agresión es posible realizar replanteamientos.

A pesar de que históricamente la Orientación haya ignorado en forma sistemática la construcción de la identidad genérica en el quehacer profesional, esta postura en los últimos años ha venido difundiéndose y debe ser asumida con claridad y conciencia disciplinaria, profesional y personal por quienes realizamos labores en este campo. Y es que el no hacerlo sería inevitablemente una invitación al desastre, afirman las orientadoras Murray y Glenn ( 1990).

\section{Acercamiento metodológico}

En este estudio se realizó un acercamiento cualitativo por medio del método fenomenológico de investigación. Esta modalidad de investigación, según Colás (1998) tiene como meta estudiar el mundo tal y como se manifiesta en el pensamiento consciente. Con la seguridad de que la descripción de las propias experiencias permite la comprensión de la subjetividad.

Este proceso investigativo inició con una revisión bibliográfica como paso necesario para asumir una postura sobre la Orientación con perspectiva perspectiva de género, lo cual, sin duda alguna, es un aporte novedoso en nuestro país.

Luego, la investigadora junto con un grupo de veinte profesionales (tres varones y diecisiete mujeres) de Orientación que laboran en la Región Educativa de San Carlos, se dieron a la tarea de dilucidar conceptos y de recoger experiencias laborales y expectativas sobre la utilización de la perspectiva de género en el quehacer orientador.

Con el propósito de recoger la información, cada profesional llenó un cuestionario (confeccionado para este propósito) con preguntas abiertas, acerca de sus concepciones, percepciones y experiencias profesionales sobre los siguientes ejes temáticos: Orientación, género, equidad, perspectiva de género y la utilización de ésta en el ejercicio profesional.

Esa información fue luego organizada en las categorías sugeridas por la misma (respetando el orden de frecuencia) para cada una de las preguntas-problema de la investigación y en una sesión de discusión grupal fue presentada, analizada y retroalimentada por el mismo grupo de profesionales.

Finalmente, para realizar el análisis general de la información recopilada se hizo uso de las técnicas de reducción de datos, exposición y elaboración de conclusiones. 


\section{Discusión de resultados}

En este apartado se presenta el análisis de la información proporcionada por el grupo de profesionales participantes en el estudio. Las preguntas de investigación planteadas dieron la pauta para estructurar la presentación del análisis realizado.

\section{A. Conceptualización de la Orientación}

En general este grupo de profesionales concibe la Orientación como una "acción o una actividad". La concretizan en un proceso o una relación de ayuda cuya finalidad se dirige, según las mujeres, a la solución de conflictos o problemas, a la dirección del desarrollo integral, y al descubrimiento y desarrollo de habilidades y destrezas específicas. Para ellas, la Orientación es:

"una relación de ayuda en la resolución de situaciones o conflictos que angustian al ser humano, se toma como prioridad a la persona, no al problema”.

"Proceso de ayuda al individuo para su realización personal, profesional, psicológica, o sea integral".

"Un proceso de ayuda a jóvenes para que descubran habilidades y destrezas".

"Proceso de ayuda al desarrollo humano: conocimiento de sí mismo, toma de decisiones y proyecto de vida".

Desde el punto de vista de los varones, la finalidad de la Orientación es el desarrollo de destrezas específicas, la elección vocacional y la formación integral. Para ellos la Orientación es:

"Formación de seres integrales en donde se expresan situaciones vividas".

"Relación de ayuda que permite al estudiante saber tomar decisiones, la elección vocacional, conocer habilidades, destrezas, valores, limitaciones y aceptarse como son".

Existe bastante similitud en la concepción sobre la Orientación que tienen las mujeres y los varones. Sin embargo, la preocupación por la atención de problemas es mencionada por las mujeres. ¿Será quizá que en los centros educativos se busca más a las mujeres para que asesoren en situaciones conflictivas de la población estudiantil? Si fuera así, sería entendible por el mismo rol asignado a las mujeres como cuidadoras y veladoras del bienestar humano.

Se nota la convivencia de diversas posturas con respecto a la misión de la Orientación, desde la atención de problemas, la toma de decisiones en general y la vocacional en particular, hasta la búsqueda de un desarrollo global de las personas. Con estas afirmaciones se confirma la conclusión a la que llegan Castro y otros (1996) con respecto a la coexistencia de diversas posturas teórico-epistemológicas en el ejercicio profesional en la actualidad.

Llama la atención el hecho de que no se visualice a la Orientación como un disciplina con su propio desarrollo histórico. De las veinte personas participantes solamente una de ellas hace alusión a la condición disciplinar de la Orientación. ¿Será que en la formación recibida no se profundiza en el rescate de esta disciplina con identidad propia?

\section{B. El binomio "sexo-género" y su importancia en el ejercicio profesional}

Este grupo de profesionales tiene, en general, claridad sobre el cómo la cultura patriarcal ha construido diferencias contrapuestas entre hombres y mujeres partiendo de la diferencia sexual.

El sexo es considerado por el grupo consultado, como el conjunto de características físicas y biológicas que determina el ser hombre o mujer. La designación genérica la relacionan con la caracterización social de los sexos, los roles, las funciones y los comportamientos determinados y aprendidos por mujeres y por hombres en la cultura machista.

En sus propias palabras el género se relaciona con:

"las características típicas o roles que se le da socialmente a cada sexo" (mujer). 
"La diferenciación de la masculinidad y la femineidad y sobre todo cómo se comporta ese hombre o esa mujer" (mujer).

"La diferencia entre lo masculino y lo femenino" (varón).

Estas afirmaciones en general, concuerdan con el pensamiento de Marta Lamas, quien afirma que "el género es una construcción simbólica, establecida entre los datos biológicos de la diferencia sexual" (Lamas, 1996, p. 12).

Este grupo de profesionales muestra preocupación por la inequidad genérica, y en general se pronuncian en pro de la equidad, la cual es equiparada a la igualdad de derechos, espacios, responsabilidades, acciones y roles entre mujeres y hombres.

Respecto a la equidad, consideran:

"la equidad es la igualdad de derechos y responsabilidades para hombres y mujeres" (mujer).

"Igualdad de condiciones en todos los espacios entre hombres y mujeres" (mujer).

"Que ambos sexos no sean como polos opuestos en su forma de convivir, que ambos tengan los mismos derechos" (mujer).

"Igualdad en el hombre y la mujer" (varón).

"Igualdad de capacidades y de oportunidades para el hombre y la mujer" (varón).

Estas afirmaciones concuerdan con el pensamiento de Marcela Lagarde, cuando ella afirma que "la igualdad significa tener las mismas oportunidades. Y que la equidad es el principio de la igual valía de las personas, que es uno de los derechos humanos universales fundamentales" (Lagarde, 1997, p. 207).

Tres de las veinte personas profesionales participantes ( 2 mujeres y 1 hombre) afirman no tener claridad ni conocimiento sobre la temática de género. Además, para la mayoría, estos conceptos no fueron temas de estudio en la formación universitaria. Los conocimientos que tienen han sido adquiridos por medio de la lectura individual y por el asesoramiento que han recibido para el desarrollo de programas específicos que demandan de su participación como profesionales.

\section{La perspectiva de género en el que- hacer orientador}

Este grupo de profesionales, en su mayoría, considera importante tomar en cuenta la realidad y las necesidades específicas de hombres y de mujeres en la acción profesional. Solamente un varón discrepa de este punto de vista.

En su mayoría consideran que existen grandes diferencias en el comportamiento de hombres y mujeres, las cuales son aprendidas, apoyadas y reforzadas por la educación en general.

En sus propias palabras:

"el hombre tiene mayor oportunidad de ascender socialmente, tiene mejores salarios, y puede asumir menos responsabilidades en la familia" (mujer).

"Todavía existe el machismo, donde se cree que la mujer es de la casa y el hombre de la calle. Con frecuencia escuchamos expresiones como: "si tú no aceptas eso, está bien, yo me busco otra", o "detrás de un gran hombre, hay una gran mujer" (mujer).

"En el presente se da el machismo aunque se pretenda creer que se ha cambiado. Se está apenas estructurando un nuevo sistema o teoría en donde se busca la igualdad de oportunidades para la mujer. Pero si hablamos de política se prefiere un hombre, las ideas masculinas son más escuchadas" (varón).

"Existen grandes diferencias en cuanto al trabajo, en las decisiones vocacionales, y en la familia para hombres y mujeres" (varón).

Las condiciones desiguales de vida para ambos sexos son bastante bien comprendidas en sus propias experiencias de vida, por este grupo de profesionales. A pesar de que la mayoría de profesionales participantes se refieren a la importancia de la equidad genérica, no tienen mucha idea sobre cómo incorporar la perspectiva de género en el ejercicio profesional. Esta misma situación la experimentan en la convivencia cotidiana en la 
familia, en el ámbito laboral y en general, en los demás espacios donde interactúan.

La comprensión de la cultura patriarcal inicia con la reflexión de la propia experiencia de vida de esta población. Para algunas mujeres profesionales esta situación ha sido vivenciada con gran conciencia desde la infancia en el ámbito familiar, y revivido en otros ámbitos, como en la escuela, el colegio y ahora en el ámbito laboral.

Con respecto a la perspectiva de género, en general este grupo de profesionales afirma no tener formación ni entrenamiento alguno, lo cual les dificulta planificar, desarrollar y evaluar su trabajo en pro de la equidad genérica. Solamente una persona se acerca al concepto al afirmar que dicha perspectiva es "un enfoque integral que busca la igualdad de género" (mujer). Sin embargo, hay quienes aportaron experiencias que muestran que en algunas actividades específicas el acercamiento generosensitivo se logra con bastante éxito. Por ejemplo:

a. Al trabajar las guías sobre sexualidad, una de las orientadoras participantes plantea que la forma en que ella las trabaja, enfatiza en las diferencias biológicas y en las conductas construidas alrededor de la definición sexual. Ella da la oportunidad al grupo de estudiantes para analizar las creencias, los mitos, las responsabilidades y la diferencia de mujeres y hombres para asumir la propia sexualidad. Ella ofrece también el espacio para la reflexión sobre la búsqueda de replanteamientos que mejoren la expresión de la sexualidad. Afirma esta orientadora que dicho tipo de actividades son bien acogidas por los grupos de estudiantes precisamente porque las jóvenes y los jóvenes tienen la oportunidad de expresar sus propias experiencias, y este tipo de espacio es muy escaso para este grupo etario.

b. Con respecto a la exploración de oportunidades de estudio y de trabajo con estudiantes de un colegio técnico, el orientador ofrece al grupo de estudiantes la oportunidad de discutir y plantear sus creencias sobre los campos de acción femeninos y masculinos. Acá se reflexiona sobre la "capacidad" y las "opciones laborales" en general. Según este profesional, tal tipo de discusión es muy dinámica y los varones expresan su preocupación por la "invasión de los espacios predefinidos como masculinos, por las mujeres que estudian carreras "no femeninas". Y es que tradicionalmente, tanto los centros educativos como la población estudiantil han reforzado los estereotipos genéricos, lo que ha ocasionado que se tienen ideas preconcebidas acerca de las carreras científicas y tecnológicas.

Tradicionalmente, las ingenierías, las ciencias físicas y las matemáticas han sido consideradas como masculinas, y los idiomas, la biología, la psicología y la sociología han sido definidas como femeninas (Radford y Holdstock 1995 en Radford, 1998). Esta caracterización tiene un peso importante en la elección de carrera ya que la presión entre "iguales" tiene una influencia central en la toma de decisiones de la población adolescente (Newton, 1986 en Radford, 1998).

Experiencias como las ya mencionadas permiten poner a discusión la realidad de las oportunidades de formación técnica y científica con la socialización genérica en esta sociedad. Según este grupo de profesionales en Orientación, son muchas las temáticas que se pueden trabajar con los estudiantes considerando la realidad genérica, y anotan las siguientes: sexualidad, oportunidades en el mundo ocupacional, mercado laboral, identidad personal, roles masculinos y femeninos, proyecto de vida, violencia doméstica, paternidad, convivencia familiar, relaciones de poder, deberes y derechos humanos. Sin embargo, para la generalización de la acción orientadora con perspectiva de género, este grupo de profesionales considera importante y necesario:

a. Capacitarse en cuanto a la teoría de género. 
b. Disponer de políticas institucionales que apoyen la incorporación de esta perspectiva en los centros educativos.

c. Que el personal docente y administrativo reciba capacitación, además de acercar más a las familias a la institución educativa para que reciban capacitación en este campo, y de esta forma coordinar el trabajo institución educativa-familia.

En general, este grupo de profesionales tiene apertura y disposición para conocer y preparse en cuanto a género y la perspectiva generosensitiva para realizar cambios significativos en el accionar profesional.

\section{Consideraciones finales}

La contribución de la Orientación como disciplina al desarrollo humano de nuestra sociedad, desde sus inicios, se verifica en sus acciones en pro de la necesidad existencial de las personas por dar sentido a sus propias vidas. Este aporte se ha tratado de brindar sin tomar en cuenta la realidad propia de hombres y mujeres con quien se trabaja, lo cual, sabemos en la actualidad, que sus consecuencias han sido la exclusión de las necesidades de las mujeres en la acción profesional.

La mayoría de profesionales que participaron en este estudio consideran importante sanar ese error histórico, sin embargo, se tiene poca claridad sobre cómo podría hacerse.

Los conceptos sobre machismo, equidad e inequidad genérica son bien comprendidos por este grupo de profesionales, no así sobre la perspectiva de género. Se manifiestan a favor de la igualdad de oportunidades y condiciones para hombres y mujeres. A pesar de que ésta sea la postura ideológica de la mayoría, no les es fácil ponerla en práctica en la vida personal y laboral. Les parece necesario incorporar las necesidades genéricas en actividades específicas sin visualizar la posibilidad de hacerlo desde la definición del propio objeto estudio de la discplina, del bagaje teórico, y en la intrervención global de la profesión.
La información y los conocimientos que este grupo de profesionales tiene sobre la temática de género, ha sido adquirida por el propio interés personal o por su requerimiento en el desarrollo de alguno de los programas institucionales. Es importante reconocer la gran disposición de estas profesinales y estos profesionales a recibir capacitación sobre la perspectiva de género y estrategias para trabajar con esta postura.

Para incorporar la perspectiva de género en las instituciones educativas, este grupo de profesionales considera importante la definición de una política educativa que con claridad asuma la condición de género como un aspecto importante en el proceso educativo. También les parece necesaria la capacitación del personal de la institución y de las familias sobre la temática.

Finalmente, los resultados de este estudio, sin duda alguna, realimentarán los planes de trabajo de profesionales en servicio y también los planes de estudio de las instancias formadoras al evidenciar algunos vacíos en la preparación de sus profesionales. Específicamente, resulta necesario reforzar la formación en cuanto la historia y el desarrollo epistemológico de esta disciplina, así como la integración de teoría de género y estrategias génerosensitivas para el desempeño profesional.

\section{Referencias bibliográficas}

Bachi, Carol. Same difference. Feminism and sexual difference. Boston: Allen \& Unwin. 1990.

Branden, Nathaniel. Cómo mejorar su autoestima. Mexico: Ediciones PAIDOS. 1991.

Bonilla, Flory Stella y Rapso, Marisol. Sentido de vida en personas mayores de 50 años. Reflexiones $N^{o}$. 59. Universidad de Costa Rica, Facultad de Ciencias Sociales. 1997. 
Castells, Manuel. La era de la información: Economía sociedad y cultura. Vol. II El poder de la identidad. México: Siglo XXI. 1999.

Campos, Alfredo. La psicoterapia no directiva. Barcelona: Herder. 1982.

Colás, María Pilar; Buendía, Leonor; Hernández, Fuensanta. Métodos de investigación en psipedagogía. España: McGrawHill Interamericana. 1998.

Clarey, Joanne y Standford, Alpheus "Female career preference and androgyny" The Vocational Guidance Quarterly. March 1982, pag. 258-264.

Corey, Gerald. Theory and practice of group counseling. California: Brooks-Cole Publishing Company. 1990.

Fernández, Juan. Género y sociedad. Madrid: Ediciones Pirámide. 1998.

Frank, Víctor. El hombre en busca de sentido España: Herder. 1984.

Hériter, Françoise Masculino-femenino: el pensamiento de la diferencia. Barcelona: Editorial Ariel S.A. 1996.

Hewitt, J. Self and society USA: Allyn and Bacon, Inc. 1988.

Lagarde, Marcela. Género y feminismo. Madrid: horas y HORAS. 1997.

Lamas, Marta. El género: la construcción sociocultural de la diferencia sexual. México: PUEG. 1996.
McMahom, M. y Patton, W. "Gender differences in childrens and adolescents' perceptions of influences on their career development. The School Counselor. Vol. 44, mayo 1997.

Ministerio de Educación Pública (MEP). Sistema Educativo Nacional: Hacia una Cultura de Equidad de Género: Plan de Acción Estratégico. San José: MEP, 1999.

Murray, Scher. y Glenn, Good. "Gender and Counseling in the twenty-first century" Journal of Counseling and Development. March-April. 1990. Vol. 68.

Radford, John. Gender and choice in education and ocupation. USA y Canadá: Routledge. 1998.

Rivas, Francisco. Manual de asesoramiento y orientación vocacional. España: Editorial Síntesis. 1995.

Ritzer, George. Sociological Theory. New York: Alfred A Knopfl. 1983.

Pearson, Judy; Turner, Lynn y Mancillas, Todd. Comunicación y género. México: ediciones PAIDOS. 1993.

Pereira, María Teresa. Orientación Educativa. Costa Rica: EUNED. 1999.

Wood, Julia. Gendered Lives. Toronto: Wadsworth Publishing Company. 1999. 